\title{
Non-stellar sources of turbulence in the interstellar medium
}

\author{
Eve C. Ostriker \\ Department of Astronomy, University of Maryland, College Park, MD 20742, USA \\ email: ostriker@astro.umd.edu
}

\begin{abstract}
Turbulence is believed to be important to star formation both within GMCs (affecting the IMF and the SFE), and on larger scales in the ISM (affecting GMC formation rates and properties). The traditional view of the ISM attributes most of the turbulent driving to stellar sources - in particular, supernovae and HII regions. However, evidence suggests that sources other than star formation must contribute significantly to large-scale turbulent driving in the diffuse ISM, thus also affecting the turbulence that GMCs inherit. I review recent investigations of alternative sources proposed for driving ISM turbulence, including thermal instabilities, the magnetorotational instability, large-scale self-gravitating modes, and instabilities in spiral shocks. I summarize results based on numerical simulations regarding the levels of turbulence that can be driven, and how these amplitudes depend on galactic conditions. This recent work shows that, under certain circumstances, very large-amplitude (trans-sonic with respect to the warm gas) motions and magnetic fields can be driven even without stellar energy inputs. Since turbulence can either trigger or limit star formation, understanding these potentially large nonstellar driving sources is crucial for a developing a theory of star formation regulation in the Milky Way and other disk galaxies.
\end{abstract}

Keywords. ISM: kinematics and dynamics, ISM: magnetic fields, methods: numerical, turbulence,magnetohydrodynamics

\section{Introduction: how does turbulence affect star formation?}

The title of this symposium links star formation with turbulence. This link may be positive, with turbulence inducing star formation, but it may also be negative. For example, turbulent large-scale velocities concentrate gas at high densities in the post-shock stagnation regions of converging flows, which encourages star formation because the gravitational collapse time decreases with increasing density. Turbulent large-scale magnetic fields also encourage star formation by transferring angular momentum out of condensations into their diffuse surroundings, enabling them to contract further. On the other hand, turbulent small-scale velocities and magnetic fields may tend to discourage star formation, by contributing to the effective pressure that counteracts self-gravity.

Within giant molecular clouds (GMCs), the star formation efficiency and rate likely depend on the overall level of turbulent compression, and the IMF may in part be established by turbulence-induced fragmentation.

In the large-scale ISM, the star formation rate and the masses of the largest bound clouds that form may depend on the turbulent velocity dispersion $\delta v$ and the turbulent Alfvén speed $\delta v_{A}=\delta B /(4 \pi \bar{\rho})^{1 / 2}$, due to turbulent contributions to an effective pressure. For isothermal gas with sound speed $c_{s}$ and total ISM surface density $\Sigma$, the rate of bound cloud formation in spiral arms scales with the Jeans time $t_{J} \equiv c_{s} /(G \Sigma)$, while the bound cloud masses scale with the Jeans mass $M_{J} \equiv c_{s}^{4} /\left(G^{2} \Sigma\right)$. Furthermore, whether or not cloud-forming (and ultimately star-forming) gravitational instabilities are possible in a sheared, rotating disk depends on the value of the Toomre parameter $Q \equiv \kappa c_{s} /(\pi G \Sigma)$. 
Here, $\kappa^{2} \equiv R^{-3} d\left(\Omega^{2} R^{4}\right) / d R$ is the squared epicyclic frequency. It is commonly believed, but has not been proven, that these results on GMC formation time scales, mass scales, and threshold criteria carry over to a turbulent medium if the sound speed $c_{s}$ is replaced by a suitable effective sound speed $c_{e f f}$ that incorporates $\delta v$ and $\delta v_{A}$. Since $\delta v$ and $\delta v_{A}$ may far exceed $c_{s}$ for a cold gas component, these effects may be extremely important.

Effects of turbulence may be important, in particular, for defining the outer edges of star-forming disks. Quirk (1972) proposed that only the portions of disks where $Q$ is sufficiently low undergo active star formation, and Kennicutt (1989), Martin \& Kennicutt (2001), and others have found that a star formation threshold criterion $Q \lesssim 1.4$ is consistent with observed data, provided that $c_{e f f} \approx 6 \mathrm{~km} \mathrm{~s}^{-1}$ is adopted. The corresponding critical gas surface density at radius $R$ for a galaxy with circular velocity $V_{c}$ is

$$
\Sigma_{c r i t} \equiv \frac{\kappa c_{e f f}}{\pi G Q_{\text {crit }}}=6 M_{\odot} \mathrm{pc}^{-2}\left(\frac{V_{c}}{200 \mathrm{~km} \mathrm{~s}^{-1}}\right)\left(\frac{c_{\text {eff }}}{6 \mathrm{~km} \mathrm{~s}^{-1}}\right)\left(\frac{R}{15 \mathrm{kpc}}\right)^{-1}\left(\frac{Q_{\text {crit }}}{1.4}\right)^{-1} \text {. }
$$

The result that $Q_{\text {crit }} \approx 1.4$ is in fact consistent with the results of numerical simulations which use isothermal gas and thus have $c_{e f f}=c_{s}$ (see e.g. the contributions of W.-T. Kim and M. Mac Low to this volume).

An alternative proposal to explain the sharp truncation of star formation in the outer parts of disks is that of Schaye (2004). He argues that star formation thresholds are defined by a transition in the available gas phases as the mean ISM pressure increases moving inward. The proposed star formation criterion is thus $P>P_{\text {min,cold }}$ where $P_{\text {min,cold }}$ is the minimum pressure at which a cold atomic phase becomes possible. For a selfgravitating gas disk (with no additional stellar or dark matter vertical gravity), the central pressure is related to the surface density by $P=\pi G \Sigma^{2} / 2$ so that the surface density at the transition from a pure-warm outer disk to a warm + cold inner disk is given by $\Sigma_{\text {trans }}=3 M_{\odot} \mathrm{pc}^{-2}\left(P_{\text {min,cold }} / 300 \mathrm{k} \mathrm{cm}^{-3} \mathrm{~K}\right)^{1 / 2}$. The value of $P_{\text {min,cold }}$ depends on UV intensity and metallicity; with a range $P_{\text {min, cold }} / \mathrm{k}=100-800 \mathrm{~cm}^{-3} \mathrm{~K}$ (Elmegreen \& Parravano 1994, Wolfire et al. 1995, Wolfire et al. 2003), the transition surface density is in the range $\Sigma_{\text {trans }}=2-5 M_{\odot} \mathrm{pc}^{-2}$.

The numerical similarity between $\Sigma_{\text {trans }}$ and $\Sigma_{\text {crit }}$ makes it difficult to judge between the gravitational instability and phase-change criteria purely based on observed values of the surface density $\Sigma_{t h}$ at the outer star-forming radii $R_{t h}$. However, other evidence suggests that the criterion $\Sigma>\Sigma_{\text {trans }}$ is not sufficient for defining a star formation threshold. In particular, Martin \& Kennicutt (2001) found that some galaxies are primarily atomic at $R_{t h}$, while other galaxies are primarily molecular. In those galaxies that are primarily molecular at $R_{t h}$, the point at which $\Sigma$ equals $\Sigma_{\text {trans }}$, and hence a cold atomic phase becomes possible, must lie at larger radius in the disk.

If the criterion $\Sigma>\Sigma_{\text {trans }}$ is necessary but not sufficient for star formation, then what happens in the radial range where $\Sigma_{t h}>\Sigma>\Sigma_{\text {trans }}$ ? If $\Sigma>\Sigma_{\text {trans }}$ in a hydrostatic (laminar) disk, a cold gas layer with a sound speed $\approx 1 \mathrm{~km} \mathrm{~s}^{-1}$ would form near the disk midplane. If $c_{\text {eff }}$ in equation (1.1) included only the thermal sound speed of $\approx 1 \mathrm{~km} \mathrm{~s}{ }^{-1}$, then $\Sigma_{\text {crit }}$ would be low, the criterion $\Sigma>\Sigma_{\text {crit }}$ would easily be met, and the cold layer would be gravitationally unstable. A very low value for $\Sigma_{\text {crit }}$, however, would be inconsistent with observed values of $\Sigma_{t h}$. This suggests that nonthermal contributions to $c_{e f f}$ raise the level significantly above $1 \mathrm{~km} \mathrm{~s}^{-1}$. If this interpretation is correct, then for self-consistency the turbulence that suppresses gravitational instability in regions with $\Sigma_{t h}>\Sigma>\Sigma_{\text {trans }}$ must have an origin not associated with star formation.

While in the outer parts of galactic disks, non-stellar turbulence sources may dominate, their contributions may also be important in inner-disk regions. In the remainder of 
this contribution, we outline potential sources of turbulence, and then discuss recent simulations that have quantified the levels of turbulence that can be produced by several different processes.

\section{Possible driving sources of ISM turbulence}

In the traditional view, turbulence in the diffuse ISM is driven mainly by supernovae. Following Spitzer (1978), the turbulent kinetic amplitude in this scenario may be estimated by equating a driving rate of turbulence with a dissipation rate. The driving rate, per unit ISM mass, is $\dot{E}_{t u r b, i n}=\epsilon_{S N} \epsilon_{S F} E_{S N} /\left(m_{S N} t_{C F}\right)$. Here $\epsilon_{S N} \approx \delta v /\left(340 \mathrm{~km} \mathrm{~s}^{-1}\right)$ is the efficiency of transferring supernova energy to the diffuse ISM (determined by the shell velocity when it becomes radiative), $\epsilon_{S F}$ is the efficiency of star formation $\left(M_{*, t o t} / M_{G M C}\right)$ per GMC lifetime, $m_{S N} \sim 200 M_{\odot}$ is the total mass in stars formed per Type II supernova, $E_{S N} \sim 10^{51}$ ergs is the total initial supernova energy, and the GMC formation time is $t_{C F} \sim t_{\text {orb }} \tau$ with $\tau \sim \min (Q, 1)$ when $Q<Q_{\text {crit }}$ and $\tau \rightarrow \infty$ when $Q>Q_{\text {crit }}$. An estimate of the overall turbulent dissipation rate in a cloudy ISM is given by $\dot{E}_{\text {turb,out }}=\delta v^{2} / t_{\text {coll }}$, where the collision time for diffuse ISM clouds is $t_{\text {coll }} \sim(H / \delta v)\left(\Sigma_{I S M} / 10 M_{\odot} \mathrm{pc}^{-2}\right)^{-1}\left(n_{H} / 10 \mathrm{~cm}^{-3}\right)\left(r_{\text {cloud }} / 10 \mathrm{pc}\right)$, or $t_{\text {coll }} \sim$ $0.1 t_{\text {orb }} Q \Sigma_{\text {cloud }} / \Sigma_{I S M}$ in terms of the Toomre parameter and the respective surface densities $\Sigma_{\text {cloud }}$ and $\Sigma_{I S M}$ of a typical cold atomic cloud and of the ISM as a whole.

Equating $\dot{E}_{t u r b, \text { in }}$ with $\dot{E}_{\text {turb,out }}$, one obtains

$$
\delta v \sim 7 \mathrm{~km} \mathrm{~s}^{-1}\left(\epsilon_{S F} / 0.05\right) \max (Q, 1)\left(\Sigma_{\text {cloud }} / 2 \Sigma_{I S M}\right) .
$$

Quantitatively, this expression agrees well with observations (e.g. Heiles \& Troland 2003) of the mean turbulent $\mathrm{H}$ I velocity dispersion. However, other considerations suggest that this agreement may in part be a coincidence. First, star formation is very intermittent it is associated with GMCs, rather than uniformly distributed throughout the H I itself. Further, these GMCs are highly concentrated in spiral arms. Yet, observations do not indicate a correlation of $\mathrm{H}$ I turbulence with star-forming regions, in terms of enhanced velocity dispersions either in arms compared to interarms, or in the inner disk compared to the outer disk (Dickey et al. 1990, van Zee \& Bryant 1999). Furthermore, as argued in $\S 1$, outer disks lack star formation, but without turbulence any cold gas layer that would develop there would be violently gravitationally unstable; thus for self-consistency other non-stellar turbulence sources are needed.

A number of potential non-stellar sources of turbulence exist; these include thermal instability, magnetic instabilities (either involving vertical magnetic buoyancy, shear and rotation combined with magnetic fields, or cosmic ray coupling to magnetic fields), largescale self-gravitational instabilities, and unsteady spiral shocks. In the following section, for several of these processes I outline the basic physical effects involved, and discuss recent results from numerical magnetohydrodynamic simulations aimed at estimating the turbulent amplitudes. Production of turbulence by interactions between cosmic rays and magnetic fields is discussed e.g. by Hanasz et al. (2004).

\section{Recent simulations of non-stellar turbulent driving}

\subsection{Thermal instability}

Under the standard heating and cooling processes for diffuse H I in galaxies, a bistable equilibrium curve results (Field, Goldsmith, \& Habing 1969, Wolfire et al. 1995, Wolfire et al. 2003). For typical mean density and pressure conditions, the atomic ISM is thermally unstable and spontaneously separates into a medium consisting of cold dense clouds 
embedded in an ambient medium of warm, diffuse gas (Field 1965). Since pressure gradients are involved in physically collecting gas as it moves from one phase to another, the thermal instability process creates random motions within the medium. Based on numerical simulations, however, this turbulence has quite low amplitude when the pressure is in the range typical of diffuse HI in the ISM, $P / k=2000-3000 \mathrm{Kcm}^{-3}$ : Piontek \& Ostriker (2004), Koyama \& Inutsuka (2006), and Brandenburg et al. (2006) all find $\delta v<0.5 \mathrm{~km} \mathrm{~s}^{-1}$. The conclusion is that thermal instability does not, by itself, significantly contribute to turbulence in the warm/cold diffuse ISM.

\subsection{Magnetorotational instability}

Rotating, magnetized disks in which the angular velocity $\Omega$ decreases outward are subject to the magnetorotational instability (MRI), which was first explored for its importance to accretion disks by Balbus \& Hawley (1991). Sellwood \& Balbus (1999) subsequently noted that MRI may be important for driving turbulence in galactic disks as well. In the MRI, magnetic tension forces mediate the transfer of angular momentum from the inner to the outer disk, with a corresponding influx of mass (see contribution of R. Piontek to this volume for further details). In three-dimensional disk models, the MRI has been shown to drive turbulence, with a quasi steady state developing after several orbits.

Galactic disks differ from accretion disks in that the gas is a multi-phase, cloudy medium. In a series of studies, Piontek \& Ostriker (2004), Piontek \& Ostriker (2005), and Piontek \& Ostriker (2006) have initiated explorations of MRI development in realistic ISM gas. One important set of questions concerns how the saturated-state turbulent amplitudes depend on the basic properties of the medium. Due to the bistable thermal equilibrium curve, the medium always evolves to a state in which the cold clouds are a factor $\sim 100$ denser and colder than the warm intercloud medium. Whereas the physical density in any parcel of gas is set by the equilibrium curve independent of $\bar{n}$, this mean density increases as the number of cold clouds per unit volume - or total ISM surface density - increases.

Based on local, non-stratified disk models, the saturated-state RMS velocity dispersion was found to vary with $\bar{n}$ following $\delta v=3 \mathrm{~km} \mathrm{~s}^{-1}\left(\bar{n} / 1 \mathrm{~cm}^{-3}\right)^{-0.77}$. This suggests that when $\bar{n} \sim 0.2 \mathrm{~cm}^{-3}$ or less, as is believed to be true in the outer parts of galaxies, turbulent velocity dispersions can become trans-sonic with respect to the warm medium (which has sound speed $\sim 7 \mathrm{~km} \mathrm{~s}^{-1}$ ). Unlike the turbulent velocity dispersion, however, the turbulent magnetic field amplitudes were found to be independent of $\bar{n}$. Instead, the magnetic field amplifies until the magnetic pressure reaches approximate equipartition with the thermal pressure: in the saturated-state, $\beta \equiv P_{t h} / P_{m a g} \sim 0.5$. This is very similar to observed magnetization levels (Heiles \& Troland (2005)): for $P / k=3000 \mathrm{~cm}^{-3}$ $\mathrm{K}$ and $B=6 \mu \mathrm{G}, \beta=0.3$.

When vertical gravity is included, differential stratification of the phases develops due to the greater buoyancy of warm compared to cold gas. If the gravity level and total gas surface density are both high (corresponding to Solar-neighborhood conditions), then a large fraction of the gas is in the cold phase, and the turbulence that is driven by MRI alone is insufficient to keep the cold clouds aloft; a thin, cold layer develops near the midplane. If the gravity and surface density are both low (corresponding to outer-galaxy conditions), on the other hand, then only a small proportion of the gas is in the cold phase. With low mean density, the MRI amplitudes are large, and a large scale height can be maintained for the cold component. For both inner- and outer-disk models, the ratio of thermal to magnetic pressure reaches realistic levels $\beta=0.3-0.6$ in the saturated state. With the low mean densities of the outer-disk model, the Alfvén speed exceeds 
$8 \mathrm{~km} \mathrm{~s}^{-1}$ even at the midplane; the velocity dispersion increases from $\sim 4 \mathrm{~km} \mathrm{~s}^{-1}$ near the midplane to twice as large at high latitudes.

The high kinetic and magnetic turbulence levels in the outer-disk model are quite interesting because they would be large enough to yield Toomre $Q$ greater than $Q_{\text {crit }}$ - and hence suppress star formation - if indeed the gravitational stability of turbulent disks follows a simple prescription based on an effective sound speed $c_{\text {eff }}$ (see $\S 1$ ). With self-gravitating simulations (now underway), it is possible to test this idea directly.

\subsection{Gravity-driven turbulence}

When the value of the Toomre $Q$ parameter is sufficiently small, disks fragment into dense clouds, which presumably further fragment into GMCs. If $Q$ is slightly larger than $Q_{c r i t}$, on the other hand, runaway collapse does not occur, but self-gravity still drives large-amplitude motions in the gas. The outer scales involved are quite large, but the turbulence is able to cascade and therefore to contribute ISM turbulence at scales less than the disk thickness as well. Using hydrodynamic simulations that also include the effects of a gravitationally-active stellar disk (modeled with N-body methods), we have found that turbulent amplitudes about half of the warm medium sound speed can be driven when $Q$ is close to $Q_{\text {crit }}$ (Kim \& Ostriker 2006b). Since observed values of $Q$ typically are close to $Q_{c r i t}$, this driving mechanism may at least intermittently - for example, in interarm regions - contribute appreciably to ISM turbulence levels.

\subsection{Unsteady spiral shocks}

Since the work of Roberts (1969), it has been known that gas entering spiral arms tends to undergo shocks (except in the immediate vicinity of corotation between the flow and the spiral pattern, $\Omega=\Omega_{p}$ ). Based on one-dimensional models (with spatial variable perpendicular to the arm), and on two-dimensional models (representing the midplane layer), these shocks are steady as long as compressions are not large enough to make the downstream gas strongly self-gravitating (e.g. Kim \& Ostriker 2002, Shetty \& Ostriker 2006). Recent work has shown, however, that in three-dimensional models the spiral shocks that form are not in general steady (Kim \& Ostriker 2006a; see also Martos \& Cox 1998, Gómez \& Cox 2002, Gómez \& Cox 2004).

To explore the shock dynamics more fully, Kim et al. (2006) performed a series of highresolution two-dimensional simulations in the radial-vertical plane. These models tested a range of spiral shock strengths and gas parameters. The typical behavior is for the shock to flap back and forth about the minimum in the spiral gravitational potential, with larger-amplitude flapping at higher $z$. The basic reason for this flapping is that, when the vertical variations in gravity are taken into account, the shock front must in general be curved in the $R-z$ plane. For a curved shock, vertical motions are excited in the gas, but the frequencies of these oscillations are not the same as the arm-to-arm crossing frequency. Gas streamlines "cross" (i.e. there is no steady flow solution), and turbulence develops and cascades to small scales. Radial and azimuthal velocity amplitudes typically exceed vertical amplitudes by a factor two, and the turbulence decays such that interarm velocities are a factor two lower than those in the post-shock arm region. For effective Mach number exceeding $\approx 4$, the total turbulent velocity dispersion is supersonic. This work should be considered preliminary since the models involve several idealizations (including adopting an isothermal equation of state), but the results suggest that spiral shocks may very significantly add to turbulence in the ISM. 


\section{Conclusion}

Stellar sources appear insufficient to power all turbulence in the ISM, while the turbulence itself is key for regulating star formation. Recent work has investigated a number of potential non-stellar turbulence sources, showing that some mechanisms are very important under certain conditions, while others are of moderate or minimal importance. In general, non-stellar turbulence sources must combine with each other and with stellar sources, although there may be some "geographic" complementarity. Understanding these interactions will be necessary for interpreting galactic turbulence observations, and for determining exactly how turbulence can both prompt and suppress star formation.

\section{Acknowledgements}

The results reported in this contribution are based on research with W.-T. Kim, C.-G. Kim, and R.A. Piontek. It is a pleasure to acknowledge numerous interesting discussions, and I am grateful for permission to present our joint work in this forum. This research has been financially supported by the National Science Foundation under AST 0507315, and by NASA under NNG05GG43G.

\section{References}

Balbus, S. A. \& Hawley, J. F. 1991, ApJ 376, 214

Brandenburg, A., Korpi, M. J. \& Mee, A. J. 2006, astro-ph/0604244

Dickey, J. M., Hanson, M. M. \& Helou, G. 1990, ApJ 352, 522

Elmegreen, B. G. \& Parravano, A. 1994, ApJ 435, L121

Field, G. B. 1965, ApJ 142, 531

Field, G.B., Goldsmith, D. W. \& Habing, H. J. 1969, ApJ 155, 149

Gómez, G. C. \& Cox, D. P. 2002, ApJ 580, 235

Gómez, G. C. \& Cox, D. P. 2004, ApJ 615, 744

Heiles, C. \& Troland, T. H. 2003, ApJ 586, 1067

Heiles, C. \& Troland, T. H. 2005, ApJ 624, 773

Hanasz, M., Kowal, G., Otmianowska-Mazur, K. \& Lesch, H. 2004, ApJ 605, L33

Kennicutt, R. C., Jr. 1989, ApJ 344, 685

Kim, W.-T. \& Ostriker, E. C. 2002, ApJ 570, 132

Kim, W.-T. \& Ostriker, E. C. 2006, ApJ 646, 213

Kim, W.-T. \& Ostriker, E. C. 2006, ApJ submitted

Kim, C.-G., Kim, W.-T. \& Ostriker, E. C. 2006, ApJ 649, L13

Kim, W.-T., Ostriker, E. C. \& Stone, J. M. 2003, ApJ 599, 1157

Koyama, H. \& Inutsuka, S.-i. 2006, astro-ph/0605528

Martin, C. L. \& Kennicutt, R. C., Jr. 2001, ApJ 555, 301

Martos, M. A. \& Cox, D. P. 1998, ApJ 509, 703

Piontek, R. A. \& Ostriker, E. C. 2004, ApJ 601, 905

Piontek, R. A. \& Ostriker, E. C. 2005, ApJ 629, 849

Piontek, R. A. \& Ostriker, E. C. 2006, ApJ submitted

Quirk, W. J. 1972, ApJ 176, L9

Roberts, W. W. 1969, ApJ 158, 123

Schaye, J. 2004, ApJ 609, 667

Sellwood, J. A. \& Balbus, S. A. 1999, ApJ 511, 660

Shetty, R. \& Ostriker, E. C. 2006, ApJ 647, 997

Spitzer, L. 1978, New York Wiley-Interscience, 1978

van Zee, Liese \& Bryant, J. 1999, AJ 118, 2172

Wolfire, M. G., Hollenbach, D., McKee, C. F., Tielens, A. G. G. M. \& Bakes, E. L. O. 1995, ApJ 443, 152

Wolfire, M. G., McKee, C. F., Hollenbach, D. \& Tielens, A. G. G. M. 2003, ApJ 587, 278 